\title{
基于空域加扰的保密无线通信统一数学模型及其 窃密方法
}

\author{
吴飞龙*，王文杰，王慧明，殷勤业
}

西安交通大学智能网络与网络安全教育部重点实验室, 西安 710049

* 通信作者. E-mail: wufeilong@stu.xjtu.edu.cn

收稿日期: 2011-08-30; 接受日期: 2011-09-28

国家自然科学基金 (批准号: 60921003, 610711258, 61172092) 资助项目

摘要 基于空域加扰的方法如人工噪声、天线阵列随机加权等可以在物理层保障无线通信的安全. 二者有着共同的物理实质,即在期望用户方向上传递保密信息, 而在其正交方向上发射人工干扰. 但 由于发射天线个数的限制, 该人工干扰在空域并非白噪声, 窃听用户可以借助接收多天线技术抑制 千扰进而解调秘密信息. 本文为上述空域加扰的两种方法建立了一致的数学模型, 并且在此基础上, 利用通信符号的有限码集特性, 提出了一种 MUSIC-like 窃听算法. 仿真结果表明, 当窃听者比发射 端有更多的天线时, 该窃听算法可以有效截获保密信息.

关键词 物理层安全人工噪声 天线阵列随机加权 有限码集 通信符号盲估计

\section{1 引言}

无线通信系统中, 由于无线信道的开放性, 信息极易被除合法用户以外的其他用户获取．而现有 的无线通信系统往往照搬有线通信系统中的方法来保障信息安全, 如在高层对信源采用密钥加密或进 行用户认证, 但这需要通信双方具有相同或相关联的密钥. 传统的跳扩频技术是另外一种保障信息安 全传输的方法, 但是需要接收方预知跳扩频序列. 显然, 不管采用哪种方法, 密钥或是跳扩频序列信息 的泄露将直接导致上述的保密通信方法失效.

近年来, 一些学者针对无线信道的特点, 提出了利用信道互易性在物理层进行保密通信的思路并 取得了一定的进展. 其基本思想为, 利用无线信道的互易性和独有性, 使得合法用户的通信质量优于非 法用户. Wyner ${ }^{[1]}$ 以及 Csiszár 等 ${ }^{[2]}$ 最早提出了窃密信道 (wiretap channel) 模型, 认为只要主信道条 件优于窃密信道, 有可能使得保密容量不为零, 这里保密容量被定义为在窃密者不能解出任何信息的 前提下, 所能达到的最大传输速率. 文献 [3] 讨论了 Gauss 白噪声信道中, 当主信道的信道容量大于窃 密信道的信道容量时, 保密容量等于两信道容量之差. 这意味着当主信道噪声干扰大于窃密信道噪声 干扰时, 保密容量为零, 即不可能实现无泄密的传输. Goel ${ }^{[4,5]}$ 从降低窃听者信号质量的角度出发, 在 基站端发射人工噪声, 降低窃听用户的信噪比, 从而增大了合法用户信道和窃密信道的容量差, 提高保 密容量. 文献 [6] 进一步研究了人工噪声方法中, 用于发射保密信息和人工噪声的功率最优分配以及 衰落信道环境下人工噪声方法遍历保密容量的下界. Li 等 ${ }^{[7,8]}$ 则提出在基站端多天线随机加权发射 
信号, 造成星座图在窃听用户处随机快速变化从而无法解调, 获得低截获率. 文献 [9] 推导了 MISOME 信道保密容量的闭式表达, 并讨论了大信噪比和天线数无穷多情况下的保密容量的极限. Mahdavifar 等在文献 [10] 中利用极化编码 ${ }^{[11]}$ 提出了一种针对二元对称离散无记忆信道的信道编码方案, 该方案 可使 Wyner 的窃密信道模型获得保密容量.

本文通过对文献 [4 8] 的研究发现, 文献中提出的人工噪声法和天线阵列随机加权法的共同实质 均是利用波束形成技术, 在合法用户方向的波束中发送保密信息: 而在其他波束中发送加性干扰, 在 保证合法用户的接收性能前提下达到降低窃听用户信噪比的效果. 据此, 本文建立了基于空域加扰保 密通信方法的统一数学模型, 将前述两种方法统一到同一的系统框架下. 基于此模型, 本文研究发现 当窃听用户使用比基站更多的天线时，有可能破解前述两种保密通信机制达到信息窃取. 由于基站天 线数目所限, 发送干扰的波束个数是有限的. 当窃听用户采用更多的天线接收时, 就有可能分离信号 和干扰, 前述两种保密通信方法的保密容量将会大大降低. 本文利用通信信号的有限码集特性, 在窃 听用户端使用一种类似 MUSIC 的通信信号盲估计算法完成了信号和干扰的分离, 达到窃取保密信息 的效果, 仿真验证了上述结论. 下文论述中, $\mathrm{T}$ 和 $\mathrm{H}$ 分别表示取矩阵转置和共轭转置, $\|\cdot\|$ 表示取向量 的 2 范数, 向量除做特殊说明外均默认为列向量.

\section{2 系统模型}

本文讨论的保密无线通信模型如图 1 所示, 基站端 Alice 向合法用户 Bob 发送信息, 但遭到非法 用户 Eve 的穷听. Alice 使用 $N_{a}$ 根天线, 合法用户 Bob 使用单天线, 钬听用户 Eve 使用 $N_{e}$ 根天线. Eve 只是被动地接收 Alice 发出的信息不做主动发射, Alice 无法获知 Eve 的信道参数, 也就很难使用 波束专门对其形成零陷或加入干扰.

Alice 到 Bob 的信道可以表示为如下一个 $N_{a}$ 维的行向量:

$$
\boldsymbol{h}_{\mathrm{AB}}=\left[h_{1}, h_{2}, \ldots, h_{i}, \ldots, h_{N_{a}}\right] .
$$

而 Alice 到 Eve 的信道可以表示为一个 $N_{e} \times N_{a}$ 的矩阵:

$$
\boldsymbol{H}_{\mathrm{AE}}=\left[\begin{array}{cccc}
h_{11} & h_{12} & \cdots & h_{1 N_{a}} \\
h_{21} & h_{22} & \cdots & h_{2 N_{a}} \\
\vdots & \vdots & \ddots & \vdots \\
h_{N_{e} 1} & h_{N_{e} 2} & \cdots & h_{N_{e} N_{a}}
\end{array}\right] .
$$

假设所有信道都是分块衰落的, 即在发送一个数据帧的间隔内, 信道是准静态的, 不同数据帧之 间信道增益系数独立同分布. Alice 利用多天线技术在时刻 $n$ 发射信号矢量 $\boldsymbol{x}(n)$, 则 Bob 和 Eve 收到 的信号分别为

$$
\begin{aligned}
& y_{B}(n)=\boldsymbol{h}_{\mathrm{AB}} \boldsymbol{x}(n)+n_{B}(n), \\
& \boldsymbol{y}_{E}(n)=\boldsymbol{H}_{\mathrm{AE}} \boldsymbol{x}(n)+\boldsymbol{n}_{E}(n),
\end{aligned}
$$

式中 $n_{B}(n)$ 和 $\boldsymbol{n}_{E}(n)$ 分别是 Bob 和 Eve 天线上接收到的加性 Gauss 噪声信号. 实现保密无线通信的 目标是要保证 Alice 发送给 Bob 的保密信息不能被 Eve 获取, 即 Eve 不能由接收到的信号 $\boldsymbol{y}_{E}(n)$ 获 得发射信号 $\boldsymbol{x}(n)$ 的准确估计. 


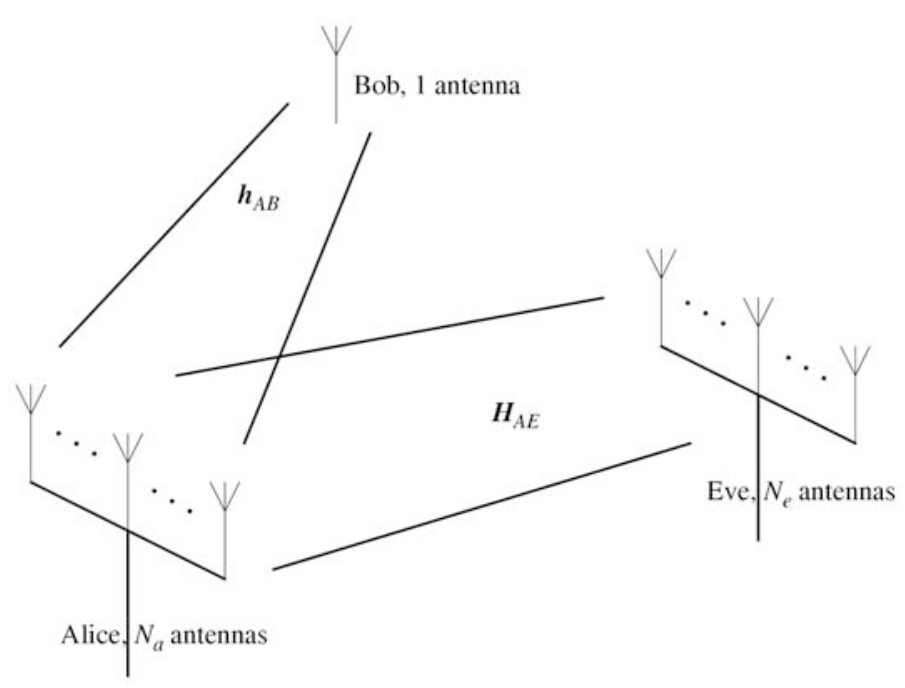

图 1 保密无线通信系统模型

Figure 1 Secure wireless communication system model

\section{3 基于空域加扰的保密通信方法的统一模型}

保密通信之前, Bob 可通过公共信道向 Alice 请求通信, 发送训练符号序列用于 Alice 完成上行 信道 $\boldsymbol{h}_{\mathrm{BA}}$ 的估计. 对于 TDD 系统, 根据信道互易原理, Alice 可以获得她到 Bob 的下行的信道 $\boldsymbol{h}_{\mathrm{AB}}$. Alice 不发射训练序列, Bob 无法估计出 $\boldsymbol{h}_{\mathrm{AB}}$, Eve 也无法获知 $\boldsymbol{H}_{\mathrm{AE}}$. 利用发射多天线技术进行空域加 扰保障通信安全的基本思路为: Alice 根据获得的合法用户信道信息 $\boldsymbol{h}_{\mathrm{AB}}$ 进行空域预均衡, 将波束主 瓣对准 Bob 使得 Bob 无需估计信道即可直接判决完成信号解调. 与此同时, Alice 利用发端多天线剩 余的自由度在与 $\boldsymbol{h}_{\mathrm{AB}}$ 正交的方向上发送人工干扰, 恶化窃听信道, 使得 Eve 的信噪比严重降低, 不能 准确解调信号. 本节首先简单介绍已提出的两种典型的空域加扰保障通信安全的方法, 然后推导二者 统一的数学模型, 最后分析这两种方法物理实质上的共性.

\section{1 人工噪声方法 $[4,5]$}

Alice 获得信道 $\boldsymbol{h}_{\mathrm{AB}}$ 后, 令 $\boldsymbol{Z}$ 为 $\boldsymbol{h}_{\mathrm{AB}}$ 零空间的一组标准正交基, 满足 $\boldsymbol{h}_{\mathrm{AB}} \boldsymbol{Z}=0$. 由于 $\boldsymbol{h}_{\mathrm{AB}}$ 的秩 为 $1, \boldsymbol{Z}$ 中含有 $N_{a}-1$ 个正交矢量, $\boldsymbol{Z}=\left[\boldsymbol{\beta}_{1}, \boldsymbol{\beta}_{2}, \ldots, \boldsymbol{\beta}_{N_{a}-1}\right]$. 在 $n$ 时刻, Alice 按如下方式在发射端产 生位于 $\boldsymbol{h}_{A B}$ 零空间的人工噪声

$$
\boldsymbol{Q}(n)=\boldsymbol{Z} \boldsymbol{a}(n)=\sum_{k=1}^{N_{a}-1} a_{k}(n) \boldsymbol{\beta}_{k},
$$

噪声向量 $\boldsymbol{Q}(n)$ 是由 $\boldsymbol{Z}$ 中列向量经线性组合得到, 组合系数 $a_{k}(n)$ 随时间独立变化且服从 Gauss 分 布 $\mathcal{C N}\left(0, \sigma_{a}^{2}\right)$. 显然, $\boldsymbol{Q}(n)$ 中元素也服从零均值 Gauss 分布, 此即人工噪声方法得名的由来. Alice 发 射叠加了人工噪声后的信号

$$
\boldsymbol{x}_{1}(n)=s(n) \boldsymbol{w}_{1}+\boldsymbol{Q}(n)=s(n) \boldsymbol{w}_{1}+\sum_{k=1}^{N_{a}-1} a_{k}(n) \boldsymbol{\beta}_{k} .
$$


表示成矩阵形式为

$$
\boldsymbol{x}_{1}(n)=\left[\boldsymbol{w}_{1}, \boldsymbol{\beta}_{1}, \ldots, \boldsymbol{\beta}_{N_{a}-1}\right]\left[\begin{array}{c}
s(n) \\
a_{1}(n) \\
\vdots \\
a_{N_{a}-1}(n)
\end{array}\right]=\boldsymbol{W} \boldsymbol{s}_{1}(n),
$$

其中 $s(n)$ 是需要保密传输的信息比特符号, $\boldsymbol{w}_{1}$ 是 $s(n)$ 的发射波束形成向量. 在文献 [5] 中为了获得 最大保密容量, $\boldsymbol{w}_{1}$ 可取

$$
\boldsymbol{w}_{1}=\frac{\boldsymbol{h}_{\mathrm{AB}}^{\mathrm{H}}}{\left\|\boldsymbol{h}_{\mathrm{AB}}\right\|} .
$$

(7) 式的发射信号模型表明, Alice 在指向 Bob 的波束 $\boldsymbol{w}_{1}$ 上发射保密信号而在窃听用户潜在的方向, 如 $\boldsymbol{\beta}_{1}, \boldsymbol{\beta}_{2}, \ldots, \boldsymbol{\beta}_{N_{a}-1}$ 上, 发送人工噪声. 由于 $\boldsymbol{h}_{\mathrm{AB}}$ 与 $\boldsymbol{\beta}_{k}$ 正交, 所以这些波束上的噪声信号不影响 Bob 的接收性能. 而 Bob 和 Eve 由于空间地理位置的不同, 无线信道 $\boldsymbol{h}_{\mathrm{AB}}$ 和 $\boldsymbol{H}_{\mathrm{AE}}$ 的相关性很小, $\boldsymbol{H}_{\mathrm{AE}}$ 中行矢量与 $\boldsymbol{\beta}_{k}$ 没有确定的正交性, 在波束 $\boldsymbol{\beta}_{1}, \boldsymbol{\beta}_{2}, \ldots, \boldsymbol{\beta}_{N_{a}-1}$ 方向上发射的人工噪声信号将严重降低 Eve 端的信噪比, 当 $N_{e}<N_{a}$ 时, Eve 无法消除干扰噪声解调保密信息. 整个通信系统的保密容量是 合法用户信道容量与窃听信道容量的差值 ${ }^{[3]}$, Eve 接收到的人工噪声使其信噪比降低, 窃听信道容量 减小, 系统保密容量得以增加.

\section{2 天线阵列随机加权方法 $[7,8]$}

当基站端 Alice 采用多天线时, 对 $n$ 时刻需要保密传输的信息符号 $s(n)$ 乘以加权系数向量 $\boldsymbol{w}_{2}(n)$, 类似发射波束成形. Alice 发射信号

$$
\boldsymbol{x}_{2}(n)=s(n) \boldsymbol{w}_{2}(n) .
$$

为了在合法用户处达到同相合并的效果, 加权向量满足如下约束:

$$
\boldsymbol{h}_{\mathrm{AB}} \boldsymbol{w}_{2}(n)=\left\|\boldsymbol{h}_{\mathrm{AB}}\right\| .
$$

该算法的出发点是使合法用户 Bob 的等效信道在一个数据帧内恒定不变, 而窃听用户的等效信道 $\boldsymbol{H}_{\mathrm{AE}} \boldsymbol{w}_{2}(n)$ 在每个码元间隔 $n$ 随机变化. Alice 可按下述方法设计 $\boldsymbol{w}_{2}(n)$ 中元素: 选定阈值 $\alpha$, 随机 选取 $\boldsymbol{h}_{\mathrm{AB}}$ 中满足 $\left|h_{i}\right|>\alpha$ 的某一元素. 假定第 $i$ 根天线上的信道增益 $h_{i}$ 被选中, $\boldsymbol{w}_{2}(n)$ 中除去与 $h_{i}$ 对应的第 $i$ 个元素 $w_{i}$ 外, 剩余的 $N_{a}-1$ 个元素 $w_{j}(j \neq i)$ 随机独立产生, 并且 $w_{j} s(n)$ 服从分布 $\mathcal{C N}\left(\mu_{w s}, \sigma_{w s}^{2}\right)$, 而权系数 $w_{i}$ 则由约束式 (10) 计算得到, 即可完成 $\boldsymbol{w}_{2}(n)$ 的设计. 这种方法可以理解为 通过时变的波束形成, 在保证 Bob 处等效信道不变的同时, 在 Eve 处生成等效的时变信道, 使得 Eve 的接收信号星座图被人为随机置乱无法解调, Alice 达到安全传输保密信息获得低截获率的目的.

\section{3 两种方法的统一表达}

上文对已提出的在物理层保障通信安全的两种算法都做了介绍, 表面上看来天线阵列随机加权法 与人工噪声的加扰机理很不相同, 下文将对两种方法通过建立统一的数学模型来揭示二者的共同的物 理实质. 对 (9) 式按以下方式处理

$$
\boldsymbol{x}_{2}(n)=s(n) \boldsymbol{w}_{2}(n)=s(n)\left[\frac{\boldsymbol{h}_{\mathrm{AB}}^{\mathrm{H}}}{\left\|\boldsymbol{h}_{\mathrm{AB}}\right\|}+\boldsymbol{w}_{2}(n)-\frac{\boldsymbol{h}_{\mathrm{AB}}^{\mathrm{H}}}{\left\|\boldsymbol{h}_{\mathrm{AB}}\right\|}\right]
$$

486 


$$
=s(n) \frac{\boldsymbol{h}_{\mathrm{AB}}^{\mathrm{H}}}{\left\|\boldsymbol{h}_{\mathrm{AB}}\right\|}+s(n)\left[\boldsymbol{w}_{2}(n)-\frac{\boldsymbol{h}_{\mathrm{AB}}^{\mathrm{H}}}{\left\|\boldsymbol{h}_{\mathrm{AB}}\right\|}\right] .
$$

在 (10) 式的约束条件下, $\boldsymbol{h}_{\mathrm{AB}}\left[\boldsymbol{w}_{2}(n)-\frac{\boldsymbol{h}_{\mathrm{AB}}^{\mathrm{H}}}{\left\|\boldsymbol{h}_{\mathrm{AB}}\right\|}\right]=0$, 因此向量 $\boldsymbol{w}_{2}(n)-\frac{\boldsymbol{h}_{\mathrm{AB}}^{\mathrm{H}}}{\left\|\boldsymbol{h}_{\mathrm{AB}}\right\|}$ 位于 $\boldsymbol{h}_{\mathrm{AB}}$ 的零空间, 进而 $s(n)\left[\boldsymbol{w}_{2}(n)-\frac{\boldsymbol{h}_{\mathrm{AB}}^{\mathrm{H}}}{\left\|\boldsymbol{h}_{\mathrm{AB}}\right\|}\right]$ 也位于 $\boldsymbol{h}_{\mathrm{AB}}$ 的零空间. 将其在 $\boldsymbol{Z}$ 上投影

$$
s(n)\left[\boldsymbol{w}_{2}(n)-\frac{\boldsymbol{h}_{\mathrm{AB}}^{\mathrm{H}}}{\left\|\boldsymbol{h}_{\mathrm{AB}}\right\|}\right]=\sum_{k=1}^{N_{a}-1} r_{k}(n) \boldsymbol{\beta}_{k},
$$

其中 $r_{k}(n)$ 为线性表出的系数, 将 (12) 式代入 (11) 式得 Alice 端发射信号为

$$
\boldsymbol{x}_{2}(n)=s(n) \frac{\boldsymbol{h}_{\mathrm{AB}}^{\mathrm{H}}}{\left\|\boldsymbol{h}_{\mathrm{AB}}\right\|}+\sum_{k=1}^{N_{a}-1} r_{k}(n) \boldsymbol{\beta}_{k},
$$

表示成矩阵形式为

$$
\boldsymbol{x}_{2}(n)=\left[\frac{\boldsymbol{h}_{\mathrm{AB}}^{\mathrm{H}}}{\left\|\boldsymbol{h}_{\mathrm{AB}}\right\|}, \quad \boldsymbol{\beta}_{1}, \ldots, \boldsymbol{\beta}_{N_{a}-1}\right]\left[\begin{array}{c}
s(n) \\
r_{1}(n) \\
\vdots \\
r_{N_{a}-1}(n)
\end{array}\right]=\tilde{\boldsymbol{W}} \boldsymbol{s}_{2}(n) .
$$

下面分析 $s_{2}(n)$ 中元素 $r_{k}(n)\left(k=1,2, \ldots, N_{a}-1\right)$ 的分布, 对 (12) 式两边同时左乘 $\boldsymbol{\beta}_{k}^{\mathrm{H}}$ 得

$$
r_{k}(n)=\boldsymbol{\beta}_{k}^{\mathrm{H}} s(n)\left[\boldsymbol{w}_{2}(n)-\frac{\boldsymbol{h}_{\mathrm{AB}}^{\mathrm{H}}}{\left\|\boldsymbol{h}_{\mathrm{AB}}\right\|}\right]=\boldsymbol{\beta}_{k}^{\mathrm{H}} s(n) \boldsymbol{w}_{2}(n),
$$

令 $\boldsymbol{\beta}_{k}=\left[b_{1}, b_{2}, \ldots, b_{N_{a}}\right]^{\mathrm{T}}, \boldsymbol{w}_{2}(n)=\left[w_{1}, w_{2}, \ldots, w_{N_{a}}\right]^{\mathrm{T}}$. 在天线阵列随机加权法中, 若 $h_{i}$ 被选中, 则根 据 (10) 式, $w_{i}=\frac{\left\|\boldsymbol{h}_{\mathrm{AB}}\right\|-\sum_{j=1, j \neq i}^{N_{a}} h_{j} w_{j}}{h_{i}}$. 将其代入 (15) 式得

$$
\begin{aligned}
r_{k}(n) & =\boldsymbol{\beta}_{k}^{\mathrm{H}} \boldsymbol{w}_{2}(n) s(n)=\frac{b_{i}^{*}\left\|\boldsymbol{h}_{\mathrm{AB}}\right\| s(n)}{h_{i}}-\frac{b_{i}^{*}}{h_{i}} \sum_{j=1, j \neq i}^{N_{a}} h_{j} w_{j} s(n)+\sum_{j=1, j \neq i}^{N_{a}} b_{j}^{*} w_{j} s(n) \\
& =\frac{b_{i}^{*}\left\|\boldsymbol{h}_{\mathrm{AB}}\right\| s(n)}{h_{i}}+\sum_{j=1, j \neq i}^{N_{a}}\left(b_{j}^{*}-\frac{b_{i}^{*}}{h_{j}} h_{i}\right) w_{j} s(n) .
\end{aligned}
$$

对于分块衰落信道, 在一个数据帧间隔内 $\boldsymbol{h}_{\mathrm{AB}}, \boldsymbol{\beta}_{k}^{\mathrm{H}}$ 中元素均保持不变, 由于 $w_{j} s(n) \sim \mathcal{C N}\left(\mu_{w s}, \sigma_{w s}^{2}\right)$, 所以 $\sum_{j=1, j \neq i}^{N_{a}}\left(b_{j}^{*}-\frac{b_{i}^{*} h_{j}}{h_{i}}\right) w_{j} s(n)$ 服从分布 $\mathcal{C N}\left(\mu_{w s} \sum_{j=1, j \neq i}^{N_{a}}\left(b_{j}^{*}-\frac{b_{i}^{*} h_{j}}{h_{i}}\right), \sigma_{w s}^{2} \sum_{j=1, j \neq i}^{N_{a}}\left|b_{j}^{*}-\frac{b_{i}^{*} h_{j}}{h_{i}}\right|^{2}\right)$. 因 此 $r_{k}(n)$ 中包含了 Gauss 噪声和泄露的信息比特分量 $\frac{b_{i}^{*}\left\|\boldsymbol{h}_{\mathrm{AB}}\right\| s(n)}{h_{i}}$. 在人工噪声方法中为了获得最大 保密容量, $\boldsymbol{w}_{1}$ 取值 $\frac{\boldsymbol{h}_{\mathrm{AB}}^{\mathrm{H}}}{\left\|\boldsymbol{h}_{\mathrm{AB}}\right\|}$. 此时对比 (7) 式和 (14) 式, 两种方法的波束成形矩阵 $\boldsymbol{W}$ 和 $\tilde{\boldsymbol{W}}$ 完全一 致, 发射信号矢量的元素均是由信息比特和 Gauss 分布的人工干扰构成. 略有不同的是天线阵列随 机加权方法中, 在其他波束方向发射的信号中泄露了一定量的保密信息 $\frac{b_{i}^{*}\left\|\boldsymbol{h}_{\mathrm{AB}}\right\| s(n)}{h_{i}}$, 当 $\left|h_{i}\right|$ 取值较大 时泄露的这部分信号能量较小. 二者共同的物理实质是 Alice 利用多天线在空域经波束方向控制矩阵 $\boldsymbol{W}=\left[\frac{\boldsymbol{h}_{\mathrm{AB}}^{\mathrm{H}}}{\left\|\boldsymbol{h}_{\mathrm{AB}}\right\|}, \boldsymbol{\beta}_{1}, \boldsymbol{\beta}_{2}, \ldots, \boldsymbol{\beta}_{N_{a}-1}\right]$ 形成 $N_{a}$ 个波束, 其中在主波束 $\frac{\boldsymbol{h}_{\mathrm{AB}}^{\mathrm{H}}}{\left\|\boldsymbol{h}_{\mathrm{AB}}\right\|}$ 方向上传递信息比特符号 $s(n)$, 在其他波束 $\boldsymbol{\beta}_{k}$ 方向传送一定量的人为随机干扰, 而随机干扰对合法用户形成零陷. 这样 Bob 能正确 解调, 而 Eve 处由于信噪比极低而无法解调获取信息. 
由于人工噪声法和天线阵列随机加权方法的相似性, 都是基于发射多天线进行空域人为干扰的思 路, 现采用统一的模型和符号来表达. Alice 发射信号

$$
\boldsymbol{x}(n)=\left[\frac{\boldsymbol{h}_{\mathrm{AB}}^{\mathrm{H}}}{\left\|\boldsymbol{h}_{\mathrm{AB}}\right\|}, \boldsymbol{\beta}_{1}, \ldots, \boldsymbol{\beta}_{N_{a}-1}\right]\left[\begin{array}{c}
s_{1}(n) \\
s_{2}(n) \\
\vdots \\
s_{N_{a}}(n)
\end{array}\right]=\boldsymbol{W} \boldsymbol{s}(n),
$$

其中 $\boldsymbol{s}(n)$ 是发射信号矢量, 元素 $s_{1}(n)$ 是需要保密传输的信息比特符号, $s_{k}(n)\left(2 \leqslant k \leqslant N_{a}\right)$ 是独立同 分布的 Gauss 干扰或是叠加了一定量保密信息的 Gauss 干扰. 当 $s(n)=s_{1}(n)$ 时, (17) 式表示人工噪 声法; 当 $s(n)=s_{2}(n)$ 时, (17) 式表示天线阵列随机加权法.

发射信号 $\boldsymbol{x}(n)$ 经无线信道传播后, 由 (3) 式和 (4) 式得到 Bob 和 Eve 分别接收到的信号为

$$
\begin{aligned}
& y_{B}=s_{1}(n)\left\|\boldsymbol{h}_{\mathrm{AB}}\right\|+n_{B}(n), \\
& \boldsymbol{y}_{E}(n)=\boldsymbol{H}_{\mathrm{AE}} \boldsymbol{W} \boldsymbol{s}(n)+\boldsymbol{n}_{E}(n) .
\end{aligned}
$$

合法用户 Bob 可以采用最大似然准则解调, 由下式获得保密信息比特

$$
\widehat{s_{1}(n)}=\arg \min _{s_{1}(n)}\left|s_{1}(n)-y_{B}(n)\right|^{2} .
$$

在 $N_{a}>N_{e}$ 时, 窃听用户 Eve 没有足够的自由度去跟踪 $s(n)$ 中干扰项的变化, 而且 $\boldsymbol{H}_{\mathrm{AE}}$ 和 $\boldsymbol{W}$ 在不同数据帧间随机变化, 无法采用 CMA 等盲均衡算法截获保密信息. 理论分析和仿真结果都表明 当 $N_{a}>N_{e}$ 时, 上述两种方法能获得一定的保密容量和低截获率 [4 8], 有效保障 Alice 到 Bob 下行链 路的信息传输安全.

\section{4 基于 MUSIC-like 盲符号估计的窃密方法}

当窃听用户使用单天线或者满足 $N_{a}>N_{e}$ 时, 上述利用基站多天线技术进行发射波束成形的保 密通信方法已被证明是有效的 [9]. 但是当网络中存在相当多的窃听用户将窃听到的信息融合等效于 $N_{e} \geqslant N_{a}$, 或者窃听用户本身的无线接收设备就满足 $N_{e} \geqslant N_{a}$, 上述基于空域加扰的通信机制将不能 提供通信安全. 其根本原因在于, 基站多天线可以生成时域的白噪声, 但受发射天线个数的限制, 就只 能生成空域的色噪声, 即定向干扰. 只要非法用户端接收天线个数大于干扰个数, 即可通过干扰抑制 方法恢复出基站发送的保密信号. 文献 [9] 对人工噪声方法的保密容量进行了分析, 其结论 “在信噪比 和天线数都趋于无穷大的极限情况下, 比值 $N_{e} / N_{a}$ 大于 1 时, 人工噪声法的保密容量存在非负上界; 比值 $N_{e} / N_{a}$ 大于 2 时, 保密容量为零” 印证了上述分析. 下面给出的一种 MUSIC-like 盲估计 ${ }^{[12]}$ 方 法可使 Eve 截获 Alice 发送的保密信息比特符号 $s_{1}(n)$.

窃听用户多天线下的接收信号向量由 (19) 式给出, 经过波束成形后的等效信道可以表示成

$$
\boldsymbol{H}=\boldsymbol{H}_{\mathrm{AE}} \boldsymbol{W} .
$$

Eve 接收到的信号 (19) 式可重写为

$$
\boldsymbol{Y}_{E}=\boldsymbol{H} \boldsymbol{S}+\boldsymbol{N}_{E}
$$


上式中

$$
\begin{aligned}
& \boldsymbol{Y}_{E}=\left[\begin{array}{llll}
\boldsymbol{y}_{E}(1) & \boldsymbol{y}_{E}(2) & \cdots & \boldsymbol{y}_{E}(K)
\end{array}\right] \\
& \boldsymbol{N}_{E}=\left[\begin{array}{llll}
\boldsymbol{n}_{E}(1) & \boldsymbol{n}_{E}(2) & \cdots & \boldsymbol{n}_{E}(K)
\end{array}\right] \\
& \boldsymbol{S}=\left[\begin{array}{llllll}
\boldsymbol{s}(1) & \boldsymbol{s}(2) & \cdots & \boldsymbol{s}(K)
\end{array}\right]=\left[\begin{array}{cccc}
s_{1}(1) & s_{1}(2) & \cdots & s_{1}(K) \\
s_{2}(1) & s_{2}(2) & \cdots & s_{2}(K) \\
\vdots & \vdots & & \vdots \\
s_{N_{a}}(1) & s_{N_{a}}(2) & \cdots & s_{N_{a}}(K)
\end{array}\right] .
\end{aligned}
$$

Eve 盲估计的目标是准确获得矩阵 $\boldsymbol{S}$ 第一行中 $K$ 个信息比特符号, 而不用去估计等效信道 $\boldsymbol{H}$. 对 (22) 式两边取转置

$$
\boldsymbol{Y}_{E}^{\mathrm{T}}=\boldsymbol{S}^{\mathrm{T}} \boldsymbol{H}^{\mathrm{T}}+\boldsymbol{N}_{E}^{\mathrm{T}}
$$

问题转化为对 $\boldsymbol{S}^{\mathrm{T}}$ 中第一列 $K$ 个元素的估计, 令 $\boldsymbol{b}=\left[s_{1}(1), s_{1}(2), \ldots, s_{1}(K)\right]^{\mathrm{T}}$ 为待估计的保密信息 向量, 对 $\boldsymbol{Y}_{E}^{\mathrm{T}}$ 进行 SVD 分解

$$
\boldsymbol{Y}_{E}^{\mathrm{T}}=\left(\begin{array}{ll}
\boldsymbol{U}_{s} & \boldsymbol{U}_{n}
\end{array}\right)\left(\begin{array}{cc}
\sum_{s} & \mathbf{0} \\
\mathbf{0} & \sum_{n}
\end{array}\right)\left(\begin{array}{c}
\boldsymbol{V}_{s}^{\mathrm{H}} \\
\boldsymbol{V}_{n}^{\mathrm{H}}
\end{array}\right) .
$$

当 $N_{e} \geqslant N_{a}$ 且 $K>N_{a}$ 时, $\boldsymbol{U}_{s}$ 张成了 $N_{a}$ 维信号子空间, $\boldsymbol{U}_{n}$ 张成了 $K-N_{a}$ 维噪声子空间并且 与 $\boldsymbol{S}^{\mathrm{T}}$ 中列向量 $\boldsymbol{b}$ 正交

$$
\boldsymbol{U}_{n}^{\mathrm{H}} \boldsymbol{b}=\mathbf{0} .
$$

为了利用 $(28)$ 式搜索 $\boldsymbol{b}$ 的估计 $\widehat{\boldsymbol{b}}$, 可以构建如下代价函数:

$$
J(\widehat{\boldsymbol{b}})=\frac{\widehat{\boldsymbol{b}}^{\mathrm{H}} \widehat{\boldsymbol{b}}}{\widehat{\boldsymbol{b}}^{\mathrm{H}} \boldsymbol{U}_{n} \boldsymbol{U}_{n}^{\mathrm{H}} \widehat{\boldsymbol{b}}} .
$$

当 $\widehat{\boldsymbol{b}}$ 恰是 Alice 发送的 $K$ 个保密信息序列 $\boldsymbol{b}$ 的准确估计时, $J(\widehat{\boldsymbol{b}})$ 取得最大. 因而, 当 Alice 的发射信 号采用 BPSK, QPSK 等有限码符号集的调制方式时, Eve 可以按 (29) 式对 $\widehat{b}$ 的所有可能情况遍历, 搜索峰值即可对 $\boldsymbol{b}$ 完成盲估计. 算法的复杂度虽然会随着 $K$ 按指数增加, 但一方面在空域加扰的保 密通信机制下, 由于 Alice 的发射天线个数有限, $K$ 值无需取得过大即可满足 $K>N_{a}$; 另一方面作为 窃听用户, 可以采用并行计算的方式减少运算时间, 有效截获保密信息.

\section{5 仿真结果}

对上文提出的 Eve 盲估计窃密方法, 给出仿真验证, 并讨论参数: Eve 接收天线数 $N_{e}$ 、信噪比 SNR, 以及 Alice 分块发送的数据帧长 $K$ 、人工干扰功率等对 Eve 误码率的影响. 这里信干比 SIR 被 定义为 Alice 发射信息比特的功率与人工干扰功率的比值, SIR 越大, 人工干扰功率相对越小. 本文分 别对前述的人工噪声和天线阵列随机加权这两种空域加扰的保密通信机制采用 MUSIC-like 解密方法 进行信息窃取仿真. 


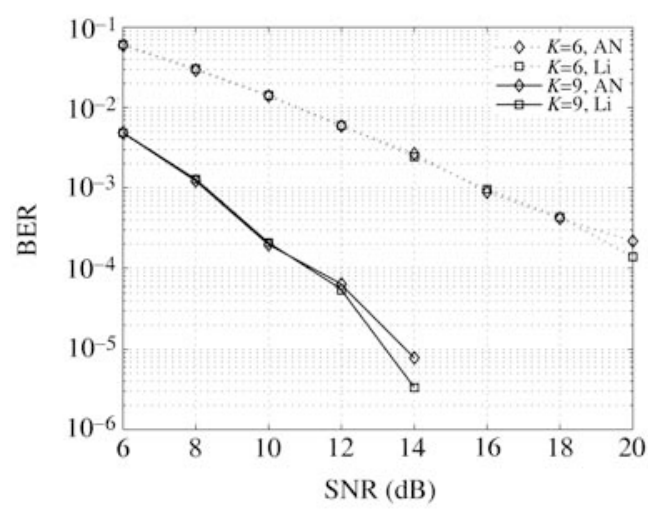

(a)

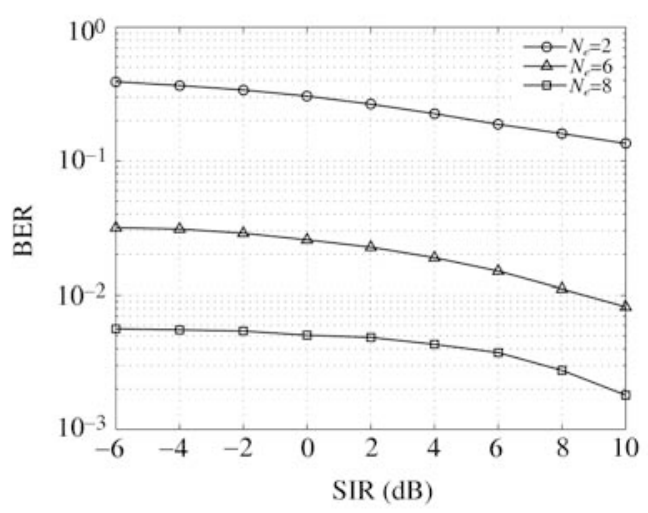

(b)

图 2 Eve 窃听算法误码率性能

Figure 2 The BER performance of Eve's wiretap algorithm. (a) The effect of SNR and block length $K$; (b) the effect of SIR and Eve's antenna number $N_{e}$

仿真实验采用平衰落瑞利信道, $\boldsymbol{H}_{\mathrm{AE}}$ 中元素是独立同分布的零均值、单位方差的复 Gauss 随机 变量, 并且在一个数据帧持续期内保持不变, 不同数据帧间独立变化. Alice 采用 4 根天线发射信号, Eve 分别采用 6 根和 8 根天线窃听, 每根窃听天线上的接收噪声相互独立且服从零均值单位方差复 Gauss 分布. 发送 100000 帧数据进行测试, 帧长 $K$ 分别取值 6 和 9, 信号调制方式为 BPSK.

当 $N_{e}=8$ 时, Alice 端等功率发送信息比特符号 $s_{1}(n)$ 和人工干扰 $s_{i}(n)\left(2 \leqslant i \leqslant N_{a}\right)$, Eve 盲估计 的性能如图 2(a) 所示. 从图中可以看出, 无论 Alice 采用人工噪声方法 (记为 AN) 还是天线阵列随机 加权法 (记为 $\mathrm{Li}$ ) 进行保密通信时, Eve 均可以采用本文提出的 MUSIC-like 算法获得较低的误码率, 且误码率性能基本一致. 另外, Eve 处信噪比越大, 误码率越小; 帧长越大, 误码率越小. 这是因为帧长 较大时能保证 $\boldsymbol{S}^{\mathrm{T}}$ 中列向量准正交, 而较小的帧长会导致 $\boldsymbol{S}^{\mathrm{T}}$ 中列向量相关而降低算法解调性能. 在 图 2(b) 中, 固定 Eve 处接收信噪比 $\mathrm{SNR}=6 \mathrm{~dB}$ 、帧长 $K=9$, 窃听用户的天线个数 $N_{e}$ 越多, 误码率 越小. 这是由于在 MUSIC-like 算法中, $N_{e}$ 相当于快拍个数, 其值越大自相关矩阵估计得越准确, 峰值 分辨率越高. 若 $N_{e}$ 不能满足 $N_{e} \geqslant N_{a}$ 的条件, Eve 的误码率会处于较高水平, MUSIC-like 算法的解 密能力就不理想. 另外, 图 2(b) 中 Eve 的误码率随人工干扰的功率减小而缓慢降低, 表明该算法的窃 密性能受人工干扰功率的影响较小, 具有一定的鲁棒性.

\section{6 结语}

实现无线保密通信, 不仅需要使用传统的数据加密和鉴权认证方法, 还应该结合无线信道本身的 特点, 在物理层保证信息的秘密传输以及密钥的分发. 本文总结了现有的两种基于空域加扰的无线保 密通信的方法, 并给出了二者统一的数学模型以及物理实质. 在此基础上, 从窃听用户角度出发, 当装 备比发射天线更多的接收天线且通信符号具有有限码集特性时, 提出一种 MUSIC-like 符号盲估计算 法可以有效截获信息, 使得现有的两种保密通信方法都失效. 因此, 若要利用多天线空域加扰的方法 实现保密通信, 就需要尽可能增加基站发射天线数, 并用较短的数据帧发送信息比特或设法去除有限 码集特性. 如何实现无线信号的保密通信以及其对立面信息窃取, 在民用尤其军事上意义重大, 新的 思路和方法有待提出. 


\title{
参考文献
}

1 Wyner A D. The wire-tap channel. Bell Syst Tech J, 1975, 54: 1355-1387

2 Csiszár I, Korner J. Broadcast channels with confidential messages. IEEE Trans Inf Theory, 1978, 24: 339-348

3 Cheong-Leung-Yan S K, Hellman M E. The Gaussian wire-tap channel. IEEE Trans Inf Theory, 1978, 24: 451-456

4 Goel S, Negi R. Secret communication in presence of colluding eavesdroppers. In: Military Communications Conference, Atlantic, 2005. 1501-1506

5 Goel S, Negi R. Guaranteeing secrecy using artificial noise. IEEE Trans Wirel Commun, 2008, 7: 2180-2189

6 Zhou X Y, McKay M R. Secure transmission with artificial noise over fading channels: achievable rate and optimal power allocation. IEEE Trans Veh Technol, 2008, 59: 2180-2189

7 Li X H, Hwu J T, Ratazzi E P. Array redundancy and diversity for wireless transmissions with low probability of interception. In: IEEE International Conference on Acoustics, Speech and Signal Processing, Toulouse 2006

8 Li X, Hwu J, Ratazzi E P. Using antenna array redundancy and channel diversity for secure wireless transmissions. J Commun, 2007, 2: 24-32

9 Khisti A, Wornell G W. Secure transmission with multiple antennas I: The MISOME wiretap channel. IEEE Trans Inf Theory, 2010, 56: 3088-3104

10 Mahdavifar H, Vardy A. Achieving the secrecy capacity of wiretap channels using polar codes. In: IEEE International Symposium on Information Theory (ISIT 2010), Austin, 2010. 13-18

11 Arikan E. Channel polarization: A method for constructing capacity achieving codes for symmetric binary-input memoryless channels. IEEE Trans Inf Theory, 2009, 55: 3051-3073

12 Li H, Wang W J, Yin Q Y. A MUSIC-like blind co-channel signals separation algorithm and its performance analysis, circuits and systems. In: IEEE International Symposium on Circuits and Systems, Taipei, 2009. 844-847

\section{A unified mathematical model for spatial scrambling based secure wireless communication and its wiretap method}

\author{
WU FeiLong*, WANG WenJie, WANG HuiMing \& YIN QinYe \\ Ministry of Education Key Lab for Intelligent Networks and Network Security, Xi'an Jiaotong University, Xi'an \\ 710049, China \\ *E-mail: wufeilong@stu.xjtu.edu.cn
}

\begin{abstract}
The spatial scrambling based wireless transmission methods, such as artificial noise and antenna array weighted randomly, can secure wireless communication at the physical layer. They have a common physical essence, that is, the private message is transmitted in the desired direction while deliberate interference is transmitted in other orthogonal directions. Unfortunately, the interference is not white in spatial domain due to the limitation of the transmit antenna number. An eavesdropper can eliminate the directional interferences by the aid of multiple antennas. In this paper, a unified mathematical model for the above physical-layer methods is established, based on which, a MUSIC-like wiretap algorithm is proposed by exploiting the finite alphabet property of communication symbols. Simulation results show that if the eavesdropper has more antennas than that of the transmitter, the proposed algorithm can intercept information effectively.
\end{abstract}

Keywords physical-layer security, artificial noise, antenna array weighted randomly, finite alphabet, communication symbols blind estimation 


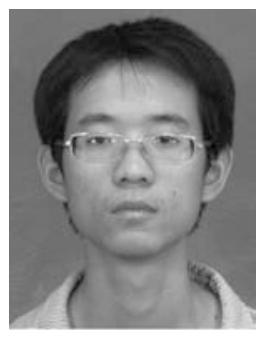

WU FeiLong was born in Anhui Province in 1988. Currently, he is a Ph.D. candidate in the Information and Communication Engineering Department at Xi'an Jiaotong University His research interests include physical layer security and key agreement in encryption system.

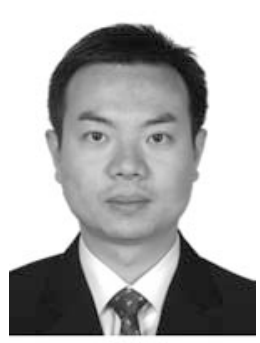

WANG HuiMing received the B.S and Ph.D. degrees, both in electrical engineering from Xi'an Jiaotong University, Xi'an in 2004 and 2010, respectively. He is currently a Lecturer at the Department of Information and Communications Engineering, Xi'an Jiaotong University, and also with the Ministry of Education Key Lab for Intelligent Networks and Network Security.

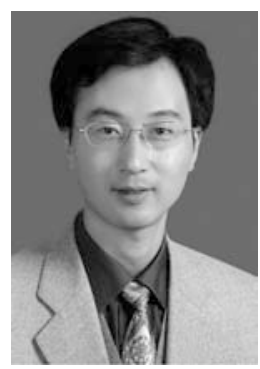

WANG WenJie received his B.S. M.S. and Ph.D. degrees in information and communication engineering from Xi'an Jiaotong University, Xi'an in 1993, 1998, and 2001, respectively. Currently, he is an Associate Professor at Xi'an Jiaotong University. His main research interests include MIMO and OFDM systems, digital signal processing, and wireless sensor networks.

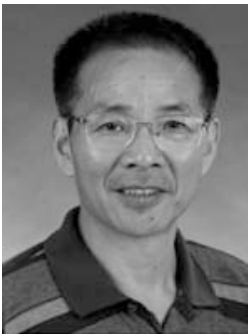

YIN QinYe received the B.S., M.S., and Ph.D. degrees in communication and electronic systems from Xi'an Jiaotong University, Xi'an in 1982, 1985 and 1989, respectively. Since 1989, he has been on the faculty at Xi'an Jiaotong University, where he is currently a Professor of Information and Communications Engineering Department and Chair of Academy Committee of School of Electronic and Information Engineering. 